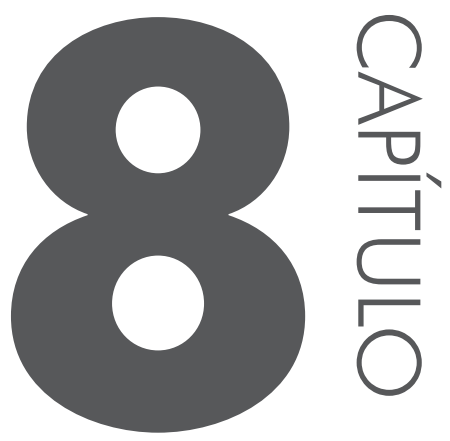

\title{
PROPOSTA DE UM MODELO DE INDICADORES DE DESEMPENHO PARA A GREEN SUPPLY CHAIN MANAGEMENT
}

Antonielli Silva Alencar ${ }^{1}$ Maico Roris Severino ${ }^{1}$

Agradecimentos: Ao CNPq e à UFG - Regional Catalão por terem concedido os recursos necessários para a realização dessa pesquisa.

Resumo: O ser humano tem causado inúmeros impactos ambientais negativos, principalmente dentro de grandes corporações, extraindo do ambiente a

1 Unidade Acadêmica Especial de Gestão e Negócios, Universidade Federal de Goiás - Regional Catalão, Catalão, Brasil. E-mails para contato: antoniellialencar@gmail.com 
matéria-prima para efetuar suas atividades de negócio. No entanto, este cenário tem sido forçado a mudanças por pressões de consumidores, legislações e/ou stakeholders. Dessa forma, empresas têm buscado cada vez mais aplicar práticas ambientalmente corretas em seus processos de gestão, como é o caso da Green Supply Chain Management (GSCM), além de avaliar o grau de utilização das práticas por meio de sistemas de medição de desempenho. Neste contexto, este trabalho teve como objetivo desenvolver uma proposta de modelo de indicadores de desempenho aplicados à GSCM. Para realização do estudo, fez-se uma revisão bibliográfica, classificando e organizando os modelos encontrados e, posteriormente, elaborou-se um novo modelo de medição de desempenho baseado nos modelos encontrados. Logo, o principal resultado obtido é a criação de um modelo de medição de desempenho mais amplo que os modelos normalmente encontrados na literatura, composto por sete grupos que ainda são divididos em atributos e indicadores qualitativos e quantitativos.

Palavras-chave: Green Supply Chain Management. Sistema de medição de desempenho. Gestão da cadeia de suprimentos.

Abstract: Humans have caused many negative environmental impacts, mainly inside big companies, extracting of the environment raw material to make their business activities. However, this scenario has been forced to change by pressures from stakeholders. In this way, companies have increasingly sought to apply environment friendly practices in their management processes, such as the Green Supply Chain Management (GSCM), evaluating the degree of utilization of practices through performance measurement systems. In this context, this study aimed to develop a proposal of a performance indicators model applied to GSCM. For this a literature review was performed, classifying and organizing the models found, and later it was elaborated a new performance measurement model based on the models found. Therefore, the main result obtained is the creation of a performance measurement model that is wider than the current models usually found in literature, compound for seven groups that are divided into qualitative and quantitative attributes and indicators.

Keywords: Green Supply Chain Management. Performance measurement. Supply chain management.

\section{INTRODUC̣ÃO}

Para Foladori (2001), a consciência de que o ser humano prejudicou a biosfera de forma radical, afetando sua própria sobrevivência, vem se consolidando desde a década de 1970. Os impactos vão desde a poluição de rios, poluição do ar de algumas cidades, extração até o esgotamento de minerais e recursos não renováveis. Devido a isso, várias empresas têm aplicado práticas ambientais, a 
fim de amenizar os impactos ambientais negativos causados. Tachizawa (2011) relata que os novos tempos são caracterizados por consumidores mais exigentes, que buscam interagir com organizações que sejam éticas, que tenham boa imagem institucional e que atuem de forma ecologicamente responsável.

Uma das formas de gerenciar o meio ambiente é através de práticas como a Green Supply Chain Management (GSCM). Uma possível tradução para o termo pode ser "gestão da cadeia de suprimentos verde". Segundo Zheng (2010), GSCM é um meio de coordenar os elos presentes numa cadeia de suprimentos desde fornecedores, fabricantes, atacadistas, varejistas até clientes finais - como uma estratégia de manter as empresas competitivas e sustentáveis. Na Figura 1 é apresentada uma esquematização do conceito de GSCM.

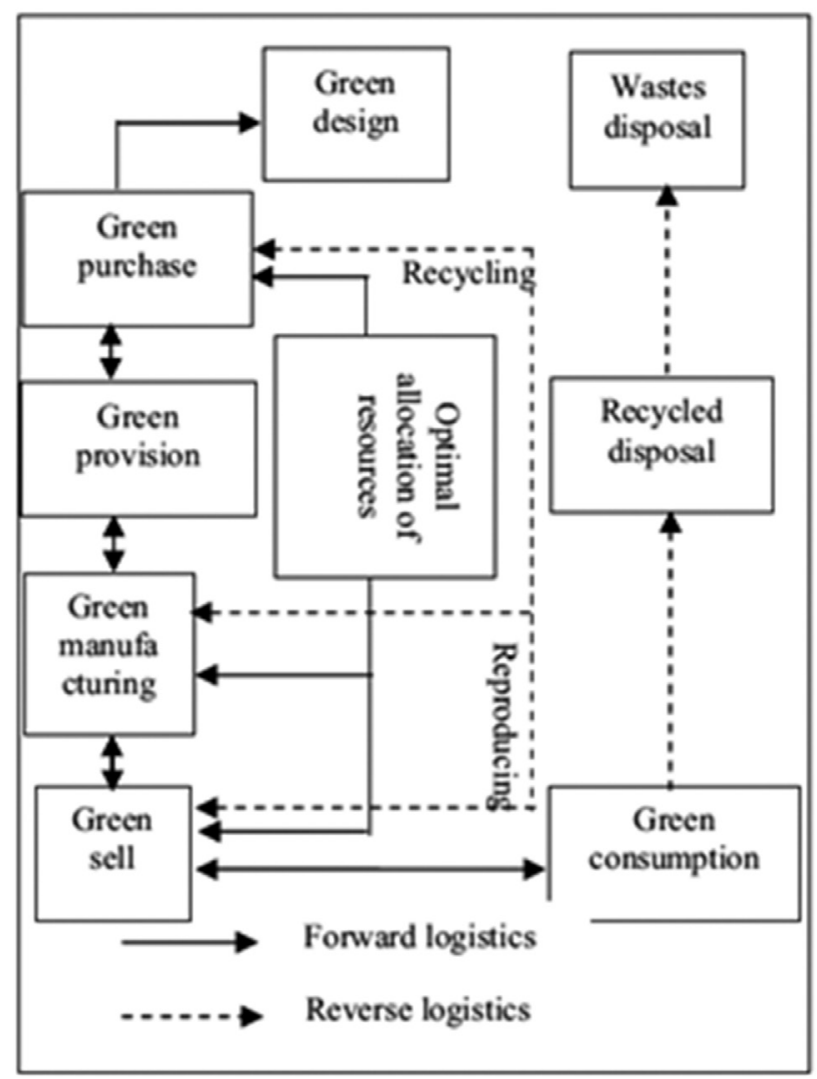

Fonte: Zheng (2010).

Figura 1. Esquematização do conceito de GSCM.

Atualmente, é de suma importância associar marcas a responsabilidade ambiental, pois além de amenizar ou eliminar problemas ambientais, as empresas se tornam bem vistas pela sociedade e ainda podem reduzir custos e aumentar a lu- 
cratividade. No entanto, faz-se necessário medir o nível de utilização das práticas por meio de sistemas de medição de desempenho.

Um sistema de medição de desempenho pode ser identificado como uma técnica utilizada para medir a eficiência e a eficácia das atividades do negócio. A eficiência está ligada à utilização dos recursos, enquanto a eficácia é responsável pela avaliação dos resultados dos processos (NEELY et al., 1995 apud XAVIER, 2008).

Percebe-se que a avaliação do desempenho da cadeia de suprimentos sustentável trará uma resposta quanto ao grau do uso das práticas de GSCM. Como a literatura traz vários modelos de sistemas de medição de desempenho, esta pesquisa tem por objetivo geral desenvolver um estudo sobre indicadores de desempenho aplicados ao GSCM, criando um abrangente modelo de medição de desempenho sustentável.

\section{METODOLOGIA}

Para a condução deste estudo foi desenvolvida uma pesquisa do tipo teórico-conceitual, feita do seguinte modo:

1) Realizou-se uma revisão bibliográfica sobre Green Supply Chain e sistemas de medição de desempenho, este último no banco de dados do Engineering Village entre os anos de 1969 até 2015, inserindo no dia 2 de abril de 2015 a combinação das palavras "Evaluation", "Performance Measurement" e "Green Supply Chain" no campo "title".

2) Foram encontrados 66 artigos, mas apenas 19 deles foram estudados por estarem disponíveis para download. Em seguida, os artigos estudados foram organizados e classificados de acordo com o número de citações e modelos de desempenho abordados.

3) Dez dos artigos estudados, que estão descritos na seção de revisão de literatura, foram selecionados para formulação de um modelo mais abrangente de medição de desempenho.

\section{REVISÃO DE LITERATURA}

\subsection{Medição de desempenho na cadeia de suprimentos}

A medição de desempenho costuma ser vista como uma atividade que mensura atributos de um processo e confere os valores mensurados com metas preestabelecidas (XAVIER, 2008). Para Kingeski (2005), na medição de desempenho de uma cadeia de suprimentos, o controle não se baseia apenas em uma empresa, mas em todas as interações ao longo de uma cadeia de processos. O sistema de medição de desempenho (SMD) mostra uma realidade às vezes complexa por causa das dificuldades em se integrar os processos das diversas empresas que cons- 
tituem a cadeia. Pires (1998) relatou que as cadeias devem adotar sistemas de avaliação de desempenho condizentes com as estratégias definidas, caso contrário os esforços de melhoria tornam-se prejudicados, devido à ausência de parâmetros comparativos, tanto em relação aos concorrentes quanto aos desejos dos clientes.

\subsection{Modelos de medição de desempenho utilizados na GSCM}

Geralmente, os modelos para desenvolvimento de um sistema de avaliação de desempenho apresentam como foco aspectos críticos dos processos, ou seja, o desenvolvimento de um sistema de medição de desempenho requer a prévia identificação do que é importante para o processo a ser avaliado (COSTA et al., 2005).

$\mathrm{Na}$ literatura há vários modelos para medição de desempenho em cadeias de suprimentos, em que cada um possui uma estrutura própria, indicadores específicos e diferentes perspectivas. O modelo proposto nesta pesquisa teve como base de desenvolvimento 10 modelos de medição de desempenho ambientais, descritos a seguir.

\subsection{Modelo de Zhu, Sarkis e Lai (2008)}

Zhu, Sarkis e Lai (2008) abordaram algumas práticas de implementação na GSCM que foram determinadas em um estudo anterior realizado pelos mesmos autores, são elas: gestão ambiental interna, compras "verdes", cooperação com consumidores incluindo requisitos ambientais, ecodesign e retorno do investimento. Além dessas práticas, os autores também relatam a existência de três variáveis de medição de resultados de desempenho: desempenho ambiental, desempenho econômico e desempenho operacional. Essas ações e variáveis são divididas em partes menores, isto é, em indicadores que podem ser mensurados quantitativa ou qualitativamente.

\subsubsection{Modelo de Olugu, Wong e Shaharoun (2011)}

Olugu, Wong e Shaharoun (2011) tinham como objetivo avaliar a cadeia de suprimentos automobilística verde e para isso foi feito um levantamento de medidas e métricas no sentido a montante da empresa focal, da própria empresa focal, a jusante e o fluxo reverso da cadeia. Foram identificados e desenvolvidos para as cadeias de frente e para trás (reversa), respectivamente, 10 medidas com 49 métricas e 6 medidas com 23 métricas.

As medidas da cadeia para frente são: compromisso com o fornecedor, custo “verde”, nível de gestão de processos, características do produto, compromisso 
de gestão, custos da cadeia de suprimentos tradicionais, capacidade de resposta, qualidade, flexibilidade e perspectiva do cliente. Já a cadeia para trás (reversa) tem como medidas: envolvimento do cliente, custo de reciclagem, recursos materiais, compromisso de gestão, eficiência de reciclagem e compromisso com o fornecedor.

\subsubsection{Modelo de Lin (2013)}

O estudo de Lin (2013) teve por objetivo analisar os fatores influentes entre os critérios de três principais práticas de GSCM, isto é, práticas, performances e pressões externas. Para lidar com a imprecisão das percepções do ser humano, o estudo utilizou teoria dos conjuntos e tomada de decisão, método de tentativa e laboratório de avaliação fuzzy para formar um modelo estrutural com objetivo de descobrir as relações de causalidade entre os critérios. As práticas avaliadas foram: compra "verde", green design, recuperação de produtos e produtos reutilizados, colaboração do fornecedor/cliente, desempenho organizacional (desempenho ambiental e desempenho econômico) e condução dos fatores externos (regulamentos e pressões dos stakeholders).

\subsubsection{Modelo de Tsang e Chiu (2013)}

O estudo de Tseng e Chiu (2013) buscou desenvolver um sistema de medição de desempenho com o intuito de selecionar um fornecedor verde para uma fabricante de placas de circuito impresso. Para tal finalidade, os autores desenvolveram um SMD com 18 critérios, dentre eles estão rentabilidade do fornecedor, relacionamento com o fornecedor, relacionamento com o consumidor, design para o ambiente e produção limpa.

\subsubsection{Modelo de Falatoonitoosi, Leman e Sorooshian (2013)}

Falatoonitoosi, Leman e Sorooshian (2013) afirmaram que o monitoramento de programas de gestão ambiental na GSCM é composto por práticas reativas e proativas, incluindo a logística reversa, reciclagem e remanufatura. No entanto, acredita-se que algo inovador na gestão da cadeia de suprimentos é a inserção de práticas como compra, embalagem e fabricação "verdes" e logística reversa. Neste sentido, os autores propuseram um modelo de medição de desempenho para seleção de fornecedores "verdes" com cinco práticas principais: desempenho organizacional (DO), logística "verde" (LV), atividades organizacionais "verdes" (AOV), proteção ambiental (PA) e avaliação de fornecedores "verdes" (AFV). Essas práticas foram então expostas como critérios de avaliação em um modelo 
de avaliação de causalidade (MAC), em que cada critério isolado está diretamente relacionado com os outros.

\subsubsection{Modelo de Wibowo (2013)}

Wibowo (2013) afirmou que é fundamental para as organizações identificar os fatores que são mais importantes para avaliar sua cadeia de suprimentos. Neste sentido, o autor fez uma ampla revisão de literatura relacionada mostrando que o problema da avaliação de desempenho da cadeia de suprimentos sustentável pode ser formulado como um entrave na tomada de decisão fuzzy multiatributo. Quatro atributos mais importantes são identificados para avaliar o desempenho de cadeias de abastecimento "verdes" em uma organização, incluindo design (C1), fabricação (C2), compras (C3) e marketing (C4).

\subsubsection{Modelo de Zhihong, Yan e He (2013)}

Zhihong, Yan e He (2013) afirmaram que um modelo de avaliação para a cadeia de suprimentos sustentável deve ser capaz de refletir uma combinação de fatores quantitativos e qualitativos. Dessa forma, os autores propuseram um SMD composto por três camadas, em que a primeira reflete o grau "verde" com base no desempenho da cadeia de suprimentos; a segunda é a camada de avaliação, isto é, tem-se um grupo pequeno de atributos que serão avaliados: rentabilidade financeira, força de mercado, avaliação de serviço ao cliente, inovação e capacidade de aprendizagem e proteção ambiental; e a terceira é formada pelos índices de cada um dos atributos.

\subsubsection{Modelo de Liv (2013)}

Liu (2013) construiu um modelo de avaliação de desempenho para a GSCM voltado a empresas de fabricação em três aspectos principais, são eles: indicadores financeiros e operacionais, indicadores ambientais e um modelo de fabricação direcionado. Este modelo de fabricação direcionado tem como objetivo avaliar os indicadores financeiros, operacionais e ambientais em cada elo da cadeia de abastecimento na indústria de manufatura. Cada indicador ainda é composto por fatores menores, tanto qualitativos quanto quantitativos.

\subsubsection{Modelo de Feng et al. (2013)}

Feng et al. (2013) empregaram o método de metanálise para resumir os índices de avaliação de desempenho para a GSCM em artigos publicados nas principais revistas chinesas entre 2005-2012. Foram encontrados 8 índices de nível I e 112 
índices de nível II. Após a combinação de índices semelhantes e remoção de índices de nível II mencionados em 15 artigos, os autores obtiveram 6 índices de nível I e 24 índices de nível II. Os seis índices de nível I são: inovação e desenvolvimento, compartilhamento de informações, desempenho ambiental, processo de negócio, serviço ao cliente e capacidade financeira. Entre os 24 índices de nível II estão à taxa de reutilização de recursos, benefício ambiental e a taxa de produção e marketing.

\subsubsection{Modelo de Diabat, Khodaverdi e Olfat (2013)}

Diabat, Khodaverdi e Olfat (2013) apresentaram um estudo sobre práticas e desempenhos do GSCM com base em uma revisão de literatura. Neste estudo deu-se destaque a sete práticas e cinco desempenhos dentro do GSCM. As práticas são: gestão ambiental interna, compras "verdes", colaboração ambiental do cliente, retorno do investimento, logística reversa, projeto para o ambiente e colaboração ambiental do fornecedor. Os desempenhos são: desempenho ambiental, desempenho econômico positivo, desempenho econômico negativo, desempenho operacional e desempenhos intangíveis.

\section{PROPOSTA}

Com base na revisão de literatura, um novo modelo de medição de desempenho foi proposto com a finalidade de englobar o máximo possível de características dos modelos já existentes, com sete grupos, como pode ser visto na Figura 2.

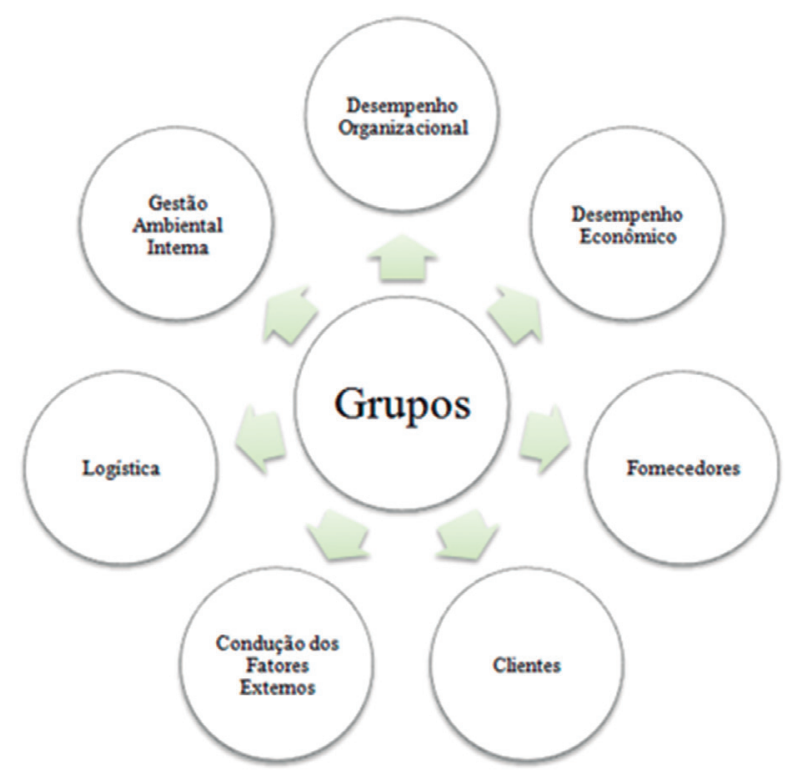

Figura 2. Grupos do modelo de medição de desempenho proposto. 
Cada um dos grupos que compõem o modelo é constituído por atributos e por indicadores. Os grupos serão apresentados com cada uma de suas partes nas próximas subseções.

\subsection{Desempenho organizacional}

No grupo desempenho organizacional, têm-se atributos e indicadores voltados tanto para a preservação ambiental quanto para o próprio desempenho do negócio em questão. Os componentes desse grupo podem ser visualizados na Tabela 1.

Tabela 1. Componentes do grupo desempenho organizacional

\section{Desempenho Organizacional}

\section{Desempenho Ambiental}

Desempenho Operacional

- Taxa de utilização de recursos e energia; - Market share;

- Taxa de redução de emissões;

- Capacidade de produção;

- Taxa de eliminação de resíduos;

- Satisfação média;

- Políticas e medidas ambientais;

- Taxa de vendas do novo produto;

- Taxa de aprovação dos sistemas de

- Taxa de redução de sucata; gestão;

- Relações com a comunidade e imagem da empresa.

- Promover a qualidade dos produtos;

- Melhorar a utilização da capacidade instalada.

\section{Processo de Negócio}

- Taxa de satisfação por pedido;

- Taxa de produção e comercialização;

- Ciclo total do pedido;

- Reconhecimento do tempo de resposta;

- Flexibilidade do produto.

\section{Inovação}

- Frequência de desenvolvimento de novos produtos;

- Taxa de sucesso dos novos produtos desenvolvidos;

- Taxa de crescimento da saída de valor de novos produtos;

- Taxa de produção de novos produtos;

- Previsão de mercado;

- Taxa de investimento em pesquisa científica;

- Proporção de pesquisadores. 


\subsection{Desempenho econômico}

Este grupo aborda atributos e indicadores que são importantes para a imagem "verde" da empresa e que também podem levar vantagem competitiva à organização. Tais elementos podem ser identificados na Tabela 2.

Tabela 2. Componentes do grupo desempenho econômico

\section{Desempenho Econômico}

\section{Rentabilidade Financeira}

- Taxa de crescimento dos lucros;

- Taxa de crescimento das vendas;

- Taxa de retorno sobre o ativo total;

- Taxa de retorno sobre o patrimônio líquido;

- Giro dos ativos e passivos com relação ao volume de negócios total em ativos fixos.

\section{Desempenho Econômico Positivo}

- Taxa da redução de custo na compra de materiais;

- Taxa de redução do custo de consumo de energia;

- Diminuição da taxa de tratamento de resíduos;

- Diminuição da taxa de descarga de resíduos;

- Redução de multa para acidentes ambientais;

- Custos relacionados a investimentos e compra de materiais ecológicos.

Desempenho Econômico Negativo Recuperação do Investimento

- Taxa de aumento de investimento;

- Taxa de aumento do custo operacional;

- Aumento dos custos para a compra de materiais ecológicos;

- Recuperação do investimento (venda) de excesso de estoques / materiais;

- Taxa de venda de sucata e materiais utilizados;

- Venda do excesso de bens de capital.

- Aumento do custo de treinamento.

\subsection{Fornecedores}

Este grupo objetiva avaliar as atitudes dos fornecedores e se há a existência de parcerias entre eles e seus clientes para colaboração ambiental. Na Tabela 3 estão dispostos os itens desse grupo. 
Tabela 3. Componentes do grupo fornecedores

\begin{tabular}{ll}
\hline \multicolumn{1}{c}{ Avaliação de Fornecedores Verdes } & \multicolumn{1}{c}{ Colaboração ambiental do Fornecedor } \\
\hline - Qualidade nos serviços; & - Educação do fornecedor sobre \\
- Uso de novas tecnologias; & questões ambientais; \\
- Nível de cooperação e atitudes & - Fornecedor de apoio aos \\
sustentáveis; & compradores "verdes"; \\
- Taxa de consumo "verde"; & Estabelecimento de programas \\
- Recursos e serviços de uso & comuns através de joint ventures de \\
relacionado a recursos naturais, & longo prazo para o desenvolvimento \\
materiais tóxicos e geração de & de inovações e soluções "verdes". \\
poluentes. & \\
\hline
\end{tabular}

\subsection{Clientes}

No grupo clientes, semelhante ao grupo fornecedores, tem-se uma avaliação da aceitação do cliente com relação aos produtos "verdes” e também se há a existência de colaboração entre fornecedor e cliente. Os atributos e indicadores desse grupo podem ser vistos na Tabela 4 .

Tabela 4. Componentes do grupo clientes

\section{Clientes}

\section{Avaliação de Atendimento ao Cliente}

- Índice de satisfação do cliente;

- Taxa de reclamações dos clientes;

- Taxa de valor para o cliente;

- Taxa de ocupação de mercado;

- Índice de oferta;

- Taxa do tempo de entrega;

- Percentual de aceitação do produto;

- Reconhecimento "verde".

\section{Colaboração Ambiental do Cliente}

- Cooperação com o cliente para o ecodesign;

- Cooperação com os clientes para uma produção mais limpa;

- Cooperação com os clientes para a embalagem "verde";

- Cooperação com os clientes para a utilização de menos energia durante o transporte do produto.

\subsection{Condução dos fatores externos}

Esse grupo está relacionado com a medição de fatores externos, como legislações que regulamentam taxas de emissões, por exemplo, e pressões das partes interessadas. Os componentes desse grupo estão organizados na Tabela 5. 
Tabela 5. Componentes do grupo condução dos fatores externos

\section{Condução dos Fatores Externos}

\section{Regulamentos}

- Taxa de resíduos e emissões;

- Nível de satisfação do cliente e comunidade;

- Cumprimento de legislações ambientais

e acordos internacionais.

\subsection{Logística}

No grupo logística, mede-se desde a redução de taxas da utilização de recursos, taxas de reciclagem, reuso e remanufatura até taxas de compartilhamento de informações. Os itens desse grupo são apresentados na Tabela 6.

Tabela 6. Componentes do grupo logística

\begin{tabular}{|c|c|}
\hline \multicolumn{2}{|c|}{ Logística } \\
\hline Logística "Verde" & Recuperação de Produtos e Produtos Reutilizados \\
\hline $\begin{array}{l}\text { - Atividades de gestão para conseguir } \\
\text { - } \text { a satisfação do cliente; } \\
\text { - Taxa de utilização dos recursos de } \\
\text { logística; } \\
\text { - Atividades sociais para a gestão da } \\
\text { logística "verde". }\end{array}$ & $\begin{array}{l}\text { - Realização de triagem dos produtos } \\
\text { reutilizáveis; } \\
\text { - Instalação de sistema de recolha } \\
\text { (reparo, remanufatura ou reciclagem). }\end{array}$ \\
\hline Logística Reversa & Compartilhamento de Informações \\
\hline $\begin{array}{l}\text { - Taxa de reutilização; } \\
\text { - Taxa de remanufatura; } \\
\text { - Taxa de reciclagem. }\end{array}$ & $\begin{array}{l}\text { - Taxa de compartilhamento de } \\
\text { informações; } \\
\text { - Taxa de pontualidade na transferência } \\
\text { de informações; } \\
\text { - Taxa de utilização da informação. }\end{array}$ \\
\hline
\end{tabular}

\subsection{Gestão ambiental interna}

Este grupo mostra a importância de se avaliar desde a fase de desenvolvimento do produto, passando pela escolha do material, impactos ambientais que o produto pode causar, utilização de recursos, requistos, normas e certificações 
ambientais até a promoção do produto. Os componentes desse grupo estão dispostos na Tabela 7.

Tabela 7. Componentes do grupo gestão ambiental interna

\section{Gestão Ambiental Interna}

\section{Green Design}

- Taxa de redução e eliminação de impactos ambientais negativos;

- Taxa de abstenção da utilização de substâncias tóxicas;

- Taxa de aumento da capacidade de inovação e economia de energia;

- Gestão dos riscos ambientais;

- Segurança do produto;

- Taxa de prevenção a poluição;

- Taxa de conservação de recursos;

- Gestão de resíduos;

- Análise do ciclo de vida.

\section{Compras "Verdes"}

- Taxa de fornecimento e compra de bens e serviços menos prejudiciais ao ambiente;

- Imagem "verde";

- Competências e habilidades de gerenciamento "verdes";

- Requisitos ambientais associados ao projeto do produto;

- Auditorias ambientais;

- Certificação ISO 14000;

- Taxa de utilização de materiais perigosos.

\section{Fabricação "Verde"}

- Taxa de fabricação de produtos que geram menos resíduos e polventes;

- Quantidade de energia gasta;

- Taxa de utilização de recursos. 


\section{Proteç̃̃o Ambiental}

- Taxa de reciclagem;

- Taxa do consumo de energia e impacto ambiental;

- Reputação ambiental;

- Taxa do consumo de água;

- Taxa da saída de valor da produção total das emissões de resíduos.

\section{CONCLUSÕES}

Este trabalho apresenta o desenvolvimento de uma proposta de modelo de medição de desempenho sustentável de acordo com os modelos estudados na revisão de literatura. O modelo proposto é composto por sete amplos grupos que ainda são divididos em atributos e indicadores. Dentre estes, há itens de medição tanto quantitativos quanto qualitativos. Assim, a partir da pesquisa realizada é possível identificar qual o desempenho das práticas sustentáveis que estão sendo utilizadas. A partir da proposta elaborada, espera-se, em trabalhos futuros, realizar a aplicação prática do modelo em casos reais, sendo assim possível avaliar comparativamente o modelo proposto com modelos reais de cadeias de suprimentos.

\section{REFERÊNCIAS}

COSTA, D. B.; FORMOSO, C. T.; LIMA, H. R.; BARTH, K. B. Sistema de indicadores para benchmarking na construção civil: Manual de Utilização. Porto Alegre: NORIE/ PPGEC/UFRGS, 2005.

DIABAT, A.; KHODAVERDI, R.; OLFAT, L. An exploration of green supply chain practices and performances in an automotive industry. The Int. J. of Adv. Manufacturing Technology, v. 68, p. 949-961, 2013.

FALATOONITOOSI, E.; LEMAN, Z.; SOROOSHIAN, S. Modeling for green supply chain evaluation. Mathematical Problems in Engineering, v. 2013, 2013.

FENG, W.; SIFENG, L.; LIJUN, Y.; WEIZHAO, L.; ZHENGYANG, Y. Research on performance evaluation system for green supply chain management based on recycled economy: taking guangxi's manufacturing industry as example. In: IEEE International Conference on Grey Systems and Intelligent Services, GSIS, 2013. Proceedings... 2013, p. 527-532.

FOLADORI, G. Limites do desenvolvimento sustentável. Campinas: Editora Unicamp, 2001. KINGESKI, L. Medição de desempenho na cadeia de suprimentos: um estudo descritivo em uma empresa automobilística. Dissertação (Mestrado em Engenharia de Produção e Sistemas) - Pontifícia Universidade Católica do Paraná, Curitiba, 2005. 133 p. LIN, R. J. Using fuzzy DEMATEL to evaluate the green supply chain management practices. Journal of Cleaner Production, v. 40, p. 32-39, 2013. 
LIU, N. Performance evaluation of green supply chain in manufacturing industry. In: IEEE International Conference on Service Operations and Logistics, and Informatics, 2013. Proceedings... 2013, p. 100-103.

OLOGU, E. U.; WONG, K. Y.; SHAHAROUN, A. M. Development of key performance measures for the automobile green supply chain. Resources, Conservation and Recycling, v. 55, p. 567-57, 2011.

PIRES, S. R. I. Gestão da cadeia de suprimentos e o modelo de consórcio modular. Revista de administração - USP, São Paulo, v. 33, n. 3, p. 5-15, 1998.

TACHIZAWA, T. Gestão ambiental e responsabilidade social corporativa: estratégias de negócio focadas na realidade brasileira. 7. ed. São Paulo: Atlas, 2011.

THIOLLENT, M. Metodologia Pesquisa-Ação. São Paulo: Autores Associados, 1988. TSENG, M-L. CHIU, A. S. F. Evaluating firm's green supply chain management in linguistic preferences. Journal of Cleaner Production, v. 40, p. 22-31, 2013.

WIBOWO, S. Fuzzy Multiattribute evaluation of green supply chain performance. In: IEEE Conference on Industrial Electronics and Applications (ICIEA), 2013, Melbourne. Proceedings... Melbourne, 2013, p. 290-295.

XAVIER, S. S. Medição de desempenho da cadeia de suprimentos: um estudo de caso em uma empresa fornecedora do setor elétrico. Dissertação (Mestrado em Engenharia de Produção) - Universidade Federal de Itajubá, Itajubá, 2008. 116 p.

ZHENG, F. Practices and research on Green Supply Chain Management in China and abroad. In: International Conference on e-Product, e-Service and e-Entertainment (ICEEE), 2010. Proceedings... Henan, 2010, p. 1-4.

ZHIHONG, W.; YAN, W.; HE, W. Performance evaluation indicator system and model construction of the green supply chain. In: International Conference on Intelligent System Design and Engineering Applications (ISDEA), 2013, Hong Kong. Proceedings... Hong Kong, 2013, p. 1042-1044.

ZHU, Q.; SARKIS, J.; LAI, K.; Confirmation of a measurement model for green supply chain management practices implementation. Int. J. Production Economics, v. 111, p. 261-273, 2008. 
